\title{
Cyclotriveratrylene-containing Porphyrins
}

Jude Deschamps, Adam Langlois, Gaël Martin, Léo Bucher, Nicolas Desbois, Claude P. Gros, and Pierre D. Harvey

\section{Supporting Information}

$\begin{array}{lll}\text { Figure S1: } & \text { HRMS ESI mass spectrum of compound } 4 & \text { p. } 2\end{array}$

$\begin{array}{lll}\text { Figure S2: } & \text { HRMS MALDI/TOF mass spectrum of 1PZn } & \text { p. } 3\end{array}$

Figure S3: $\quad$ HRMS MALDI/TOF mass spectrum of $\mathbf{2 P Z n} \quad$ p. 4

Figure S4: HRMS MALDI/TOF mass spectrum of compound 3PZn $\quad$ p. 5

Figure S5: HRMS MALDI/TOF mass spectrum of compound 6PZn $\quad$ p. 6

Figure S6: $\quad$ MS MALDI/TOF mass spectrum of compound 6PZn $\quad$ p. 7

$\begin{array}{lll}\text { Figure S7: } & { }^{1} \mathrm{H} \text { NMR spectrum of } \mathbf{4}\left(300 \mathrm{MHz}, \mathrm{CDCl}_{3}\right) & \text { p. } 8\end{array}$

Figure S8: $\quad{ }^{1} \mathrm{H}$ NMR spectrum of $5\left(300 \mathrm{MHz}^{\left.-\mathrm{CDCl}_{3}\right)}\right.$ p. 9

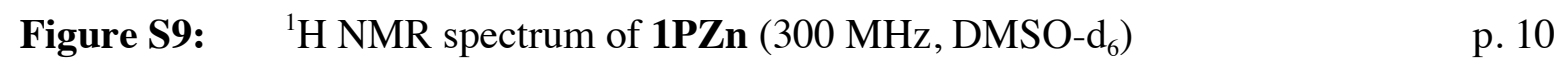

Figure S10: $\quad{ }^{1} \mathrm{H}$ NMR spectrum of $\mathbf{2 P Z n}\left(300 \mathrm{MHz}, \mathrm{DMSO}-\mathrm{d}_{6}\right) \quad$ p. 11

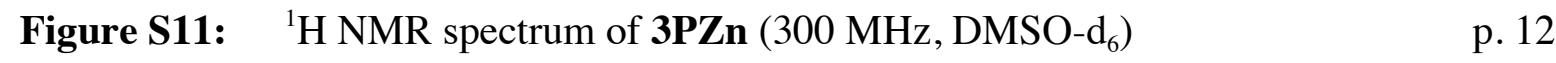

Figure S12: $\quad{ }^{1} \mathrm{H}$ NMR spectrum of 6 PZn $\left(300 \mathrm{MHz}, \mathrm{THF}_{8}\right) \quad$ p. 13

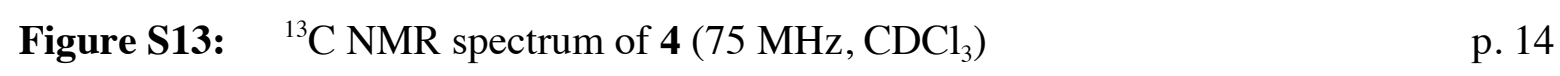

Figure S14: ${ }^{13} \mathrm{C}$ NMR spectrum of $5\left(75 \mathrm{MHz}, \mathrm{CDCl}_{3}\right) \quad$ p. 15

$\begin{array}{lll}\text { Figure S15: } & \text { Analytical HPLC of 1PZn } & \text { p. } 16\end{array}$

$\begin{array}{ll}\text { Figure S16: Analytical HPLC of 2PZn } & \text { p. } 17\end{array}$

$\begin{array}{lll}\text { Figure S17: } & \text { Analytical HPLC of 3PZn } & \text { p. } 18\end{array}$

$\begin{array}{lll}\text { Figure S18: } & \text { Analytical HPLC of 6PZn } & \text { p. } 19\end{array}$

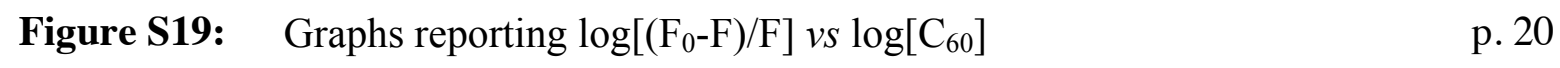

Figure S20: $\quad$ Computer modeling of $2 \mathbf{P Z n}$ and corresponding $2 \mathbf{P Z n} \cdots \mathrm{C}_{60} \quad$ p. 21

Figure S21: Computer modeling of $\mathbf{3 P Z n}$ and corresponding $3 \mathbf{P Z n} \cdots \mathrm{C}_{60} \quad$ p. 22 


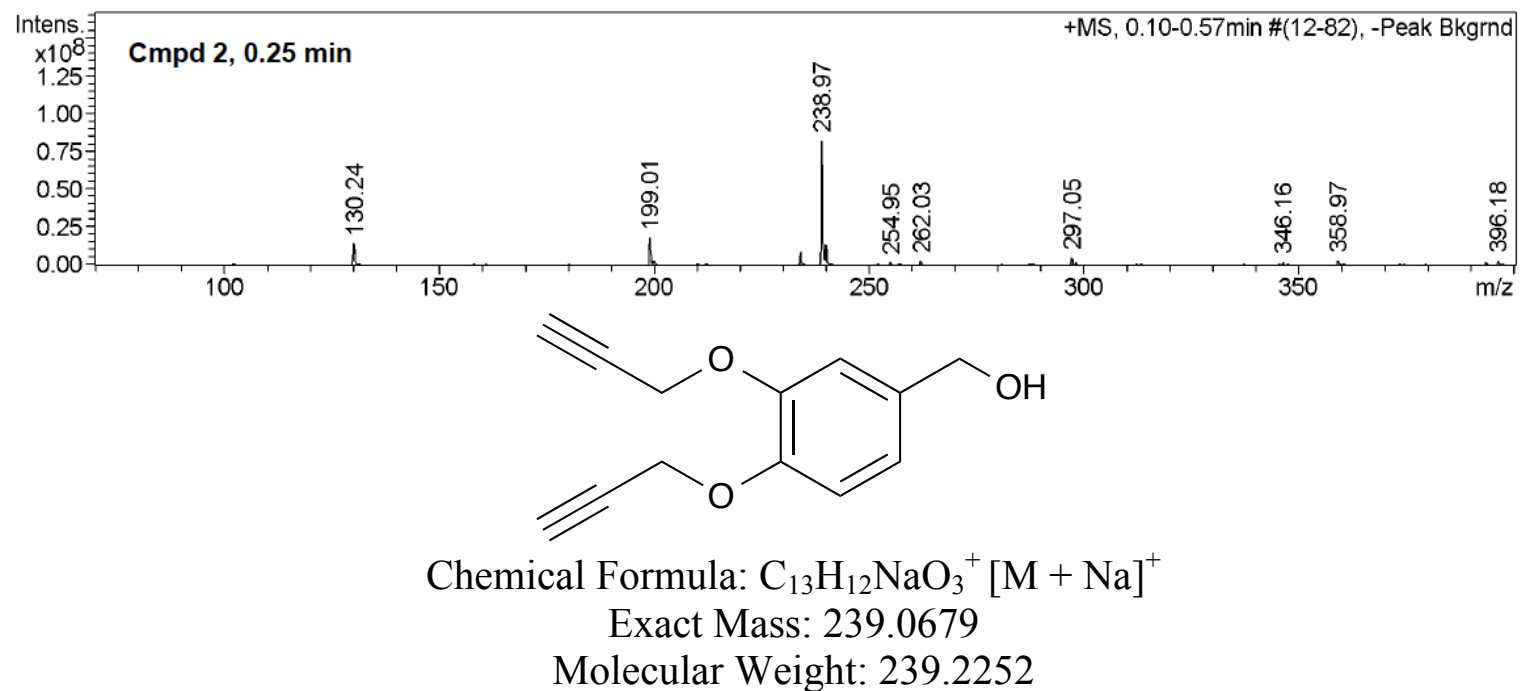

Figure S1: HRMS ESI mass spectrum of compound 4 


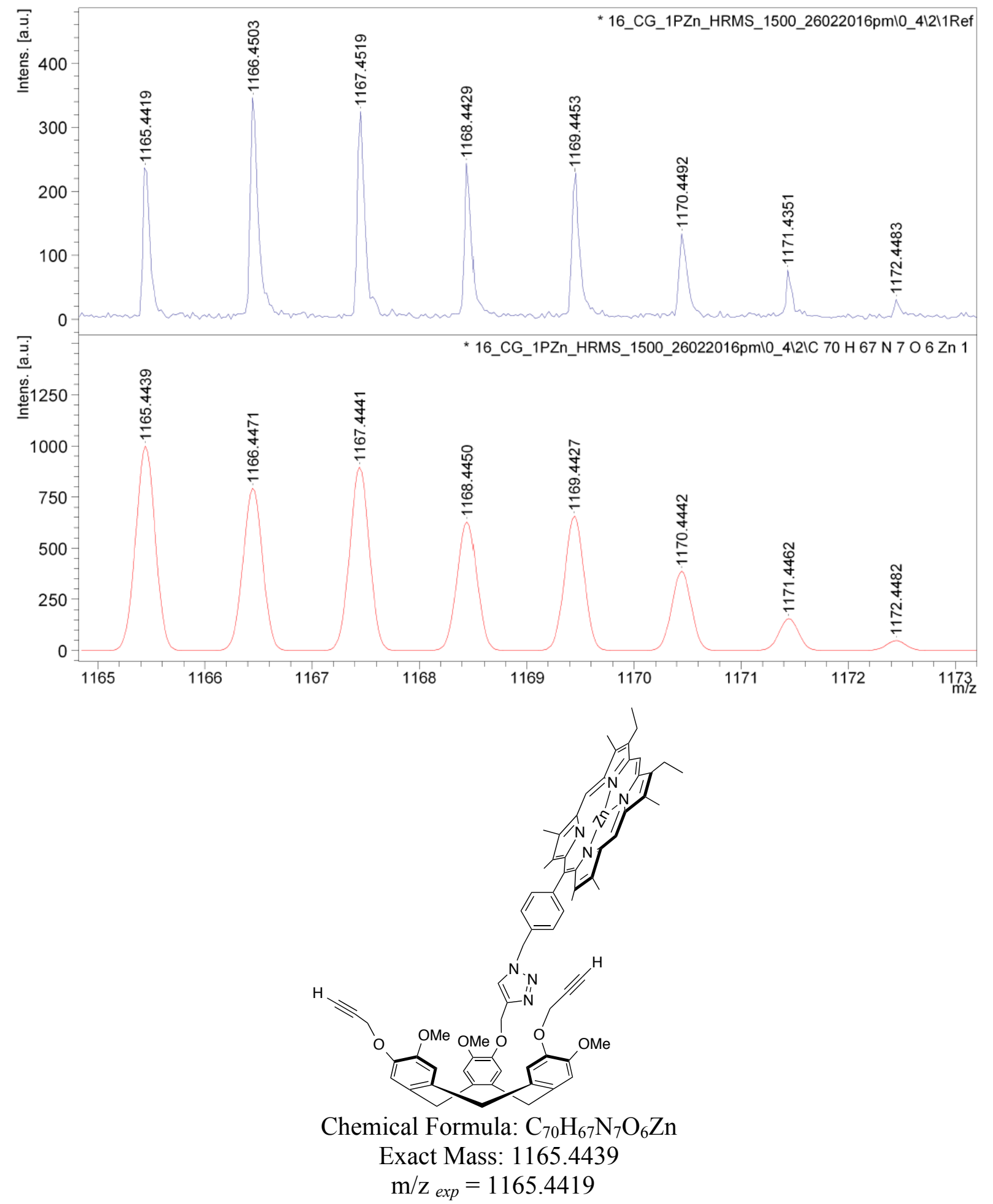

Figure S2: HRMS MALDI/TOF mass spectrum of 1PZn 


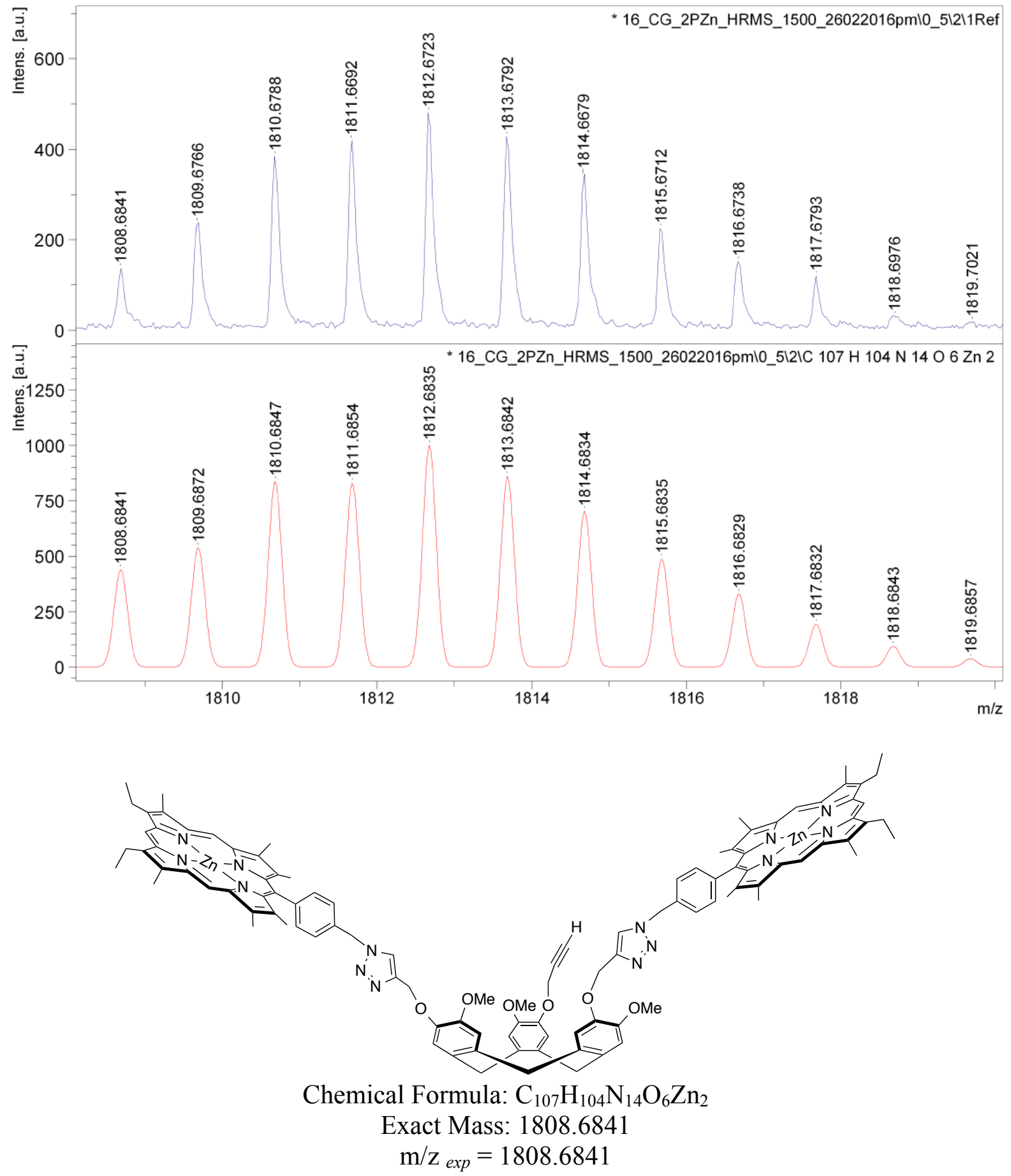

Figure S3: HRMS MALDI/TOF mass spectrum of 2PZn 

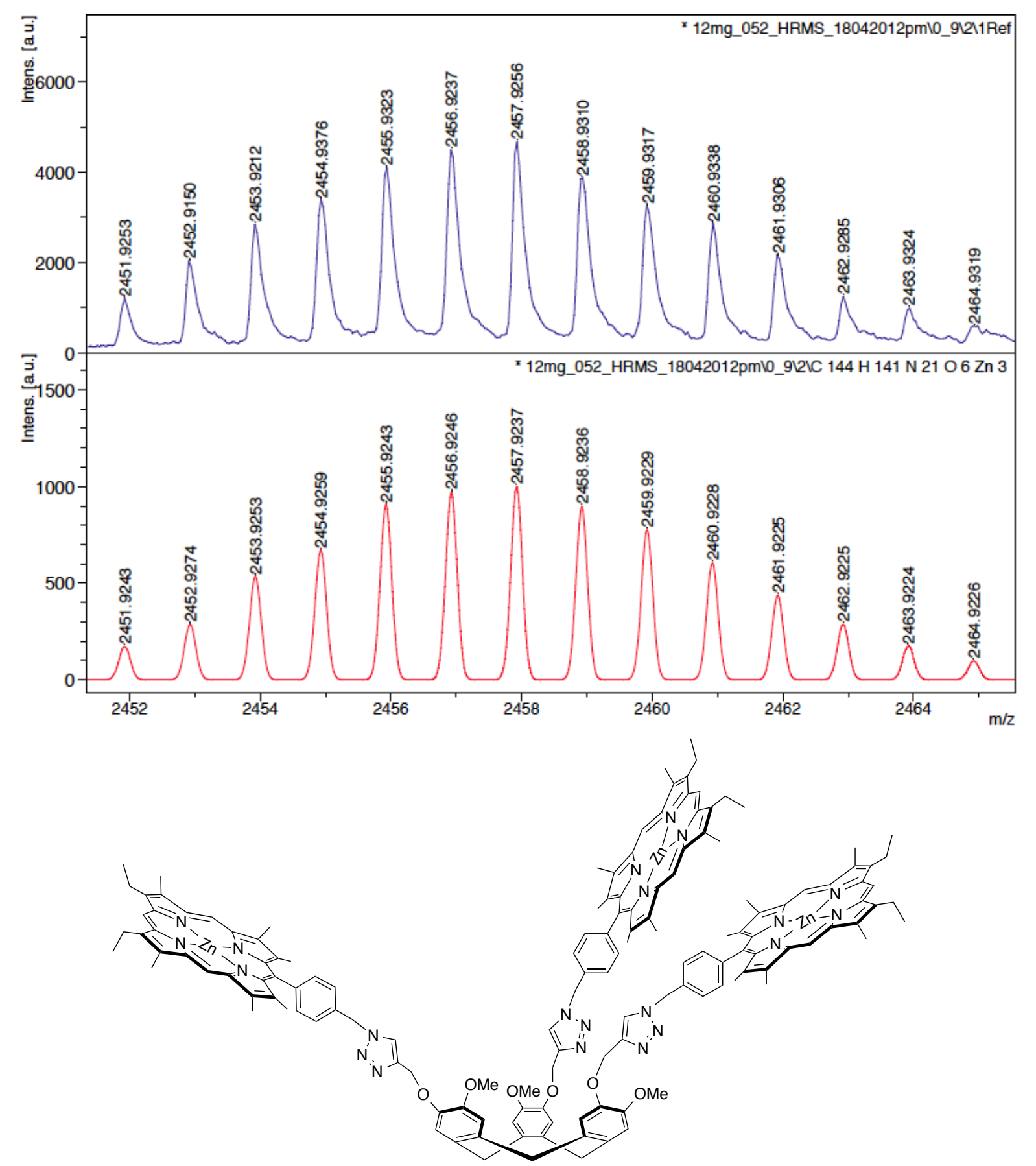

Chemical Formula: $\mathrm{C}_{144} \mathrm{H}_{141} \mathrm{~N}_{21} \mathrm{O}_{6} \mathrm{Zn}_{3}$

Exact Mass: 2451.9243

$\mathrm{m} / \mathrm{z}_{\text {exp }}=2451.9253$

Figure S4: HRMS MALDI/TOF mass spectrum of compound 3PZn 

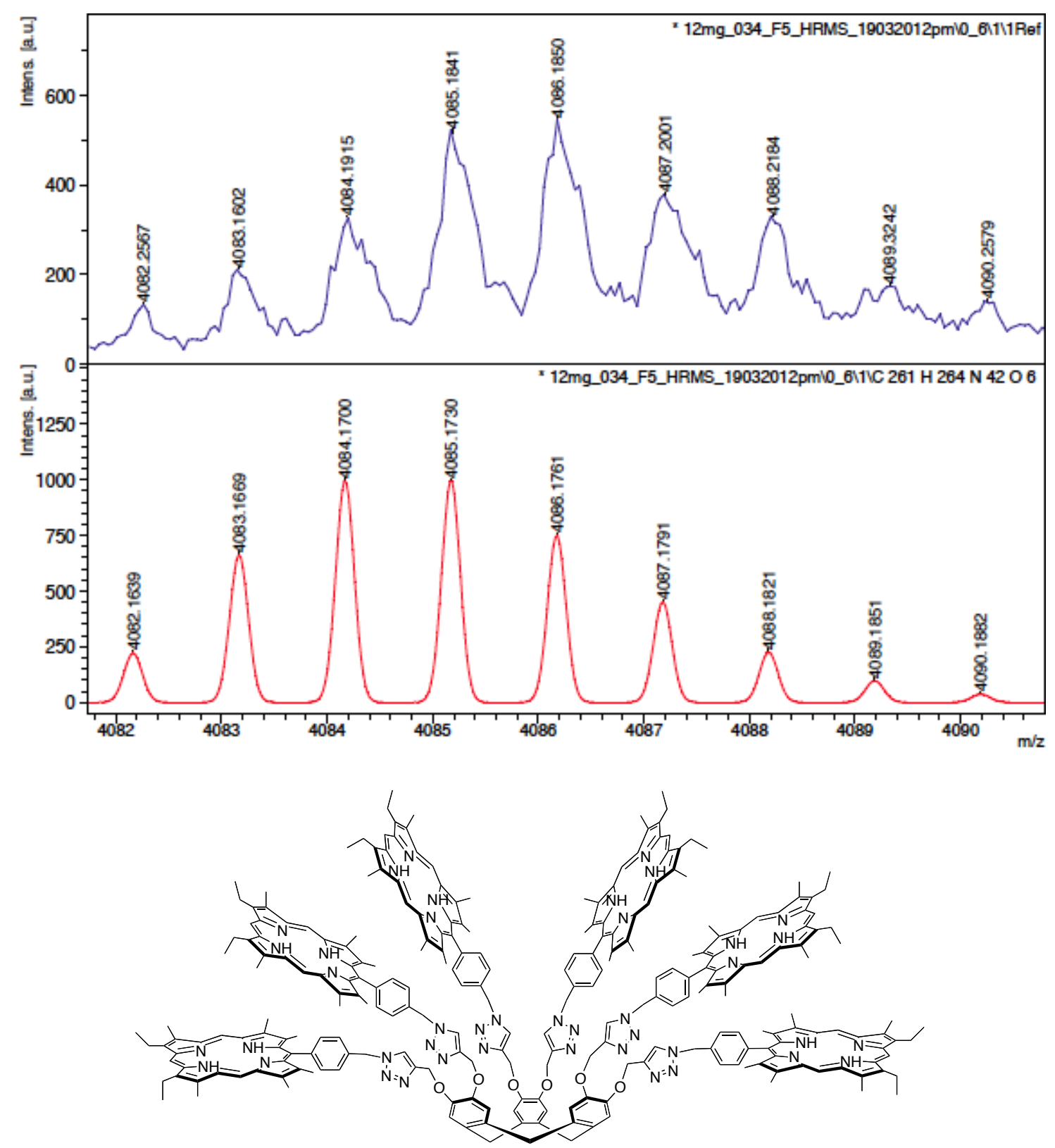

Chemical Formula: $\mathrm{C}_{261} \mathrm{H}_{264} \mathrm{~N}_{42} \mathrm{O}_{6}$ *

Exact Mass: 4082.1644 g. $\mathrm{mol}^{-1}$

$\mathrm{m} / \mathrm{z}_{\text {exp }}=4082.2657 \mathrm{~g} \cdot \mathrm{mol}^{-1}$

*The 6PZn form didn't fly in the TOF analyzer (despite different conditions used for the registration of the MS spectrum). We thus have to used TFA to remove the six Zinc metal ions and to help ionisation by MALDI mode.

Figure S5: HRMS MALDI/TOF mass spectrum of compound 6PZn 

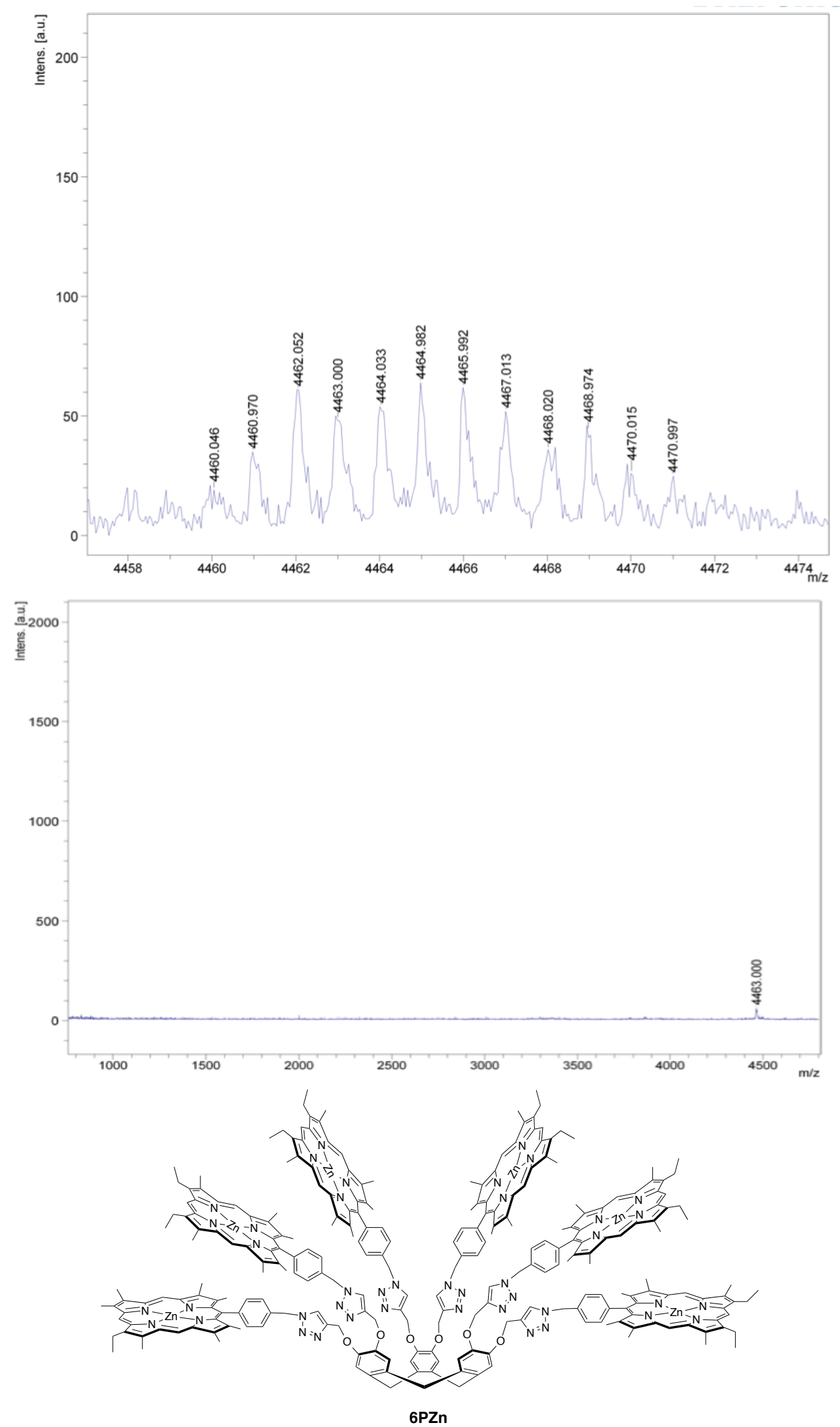

Chemical Formula: $\mathrm{C}_{261} \mathrm{H}_{252} \mathrm{~N}_{42} \mathrm{O}_{6} \mathrm{Zn}_{6}$

Exact Mass: 4453.6454

Molecular Weight: 4465.4550

Figure S6: MS MALDI/TOF mass spectrum of compound 6PZn 


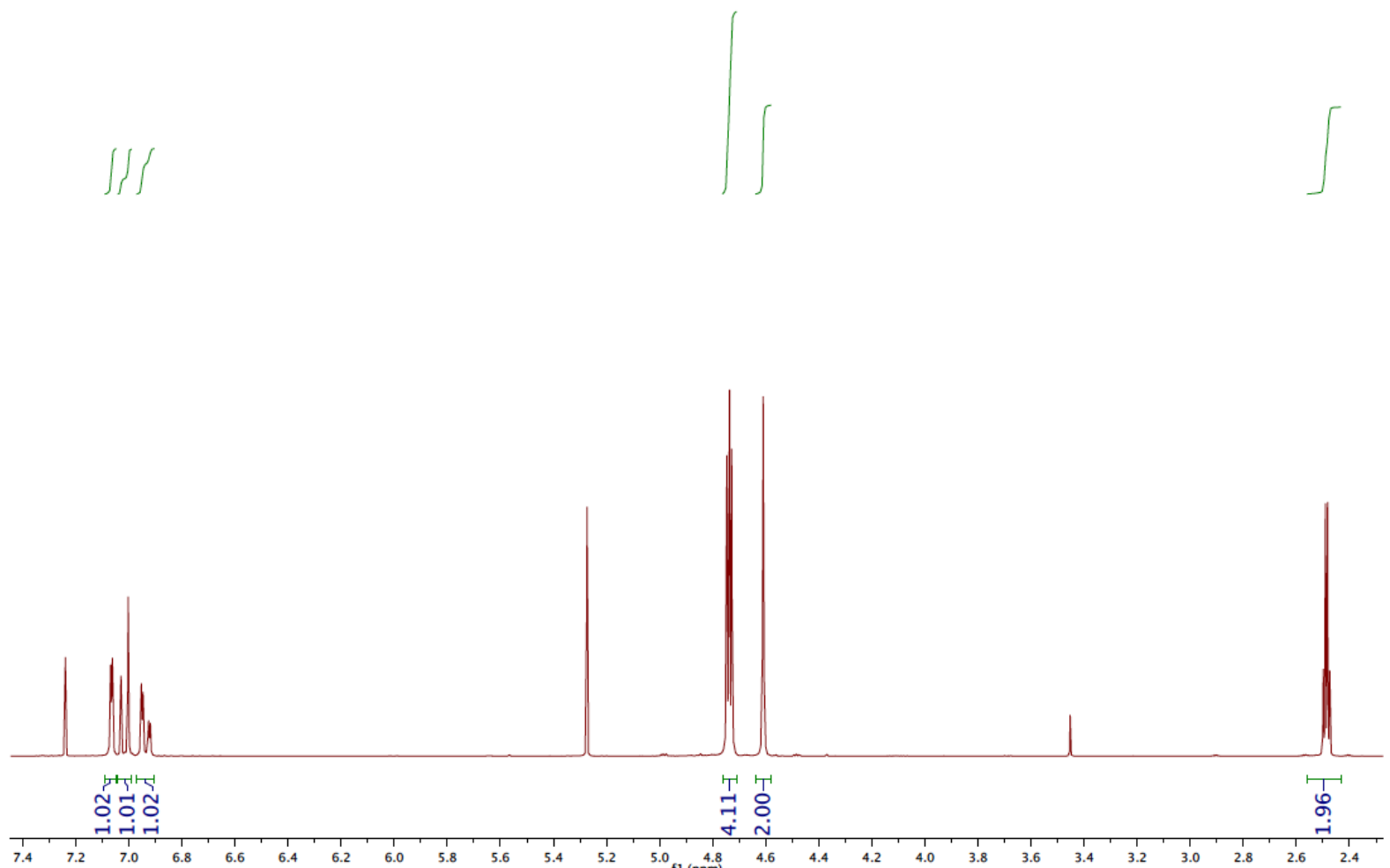

Figure S7: ${ }^{1} \mathrm{H}$ NMR spectrum of $4\left(300 \mathrm{MHz}, \mathrm{CDCl}_{3}\right)$ 

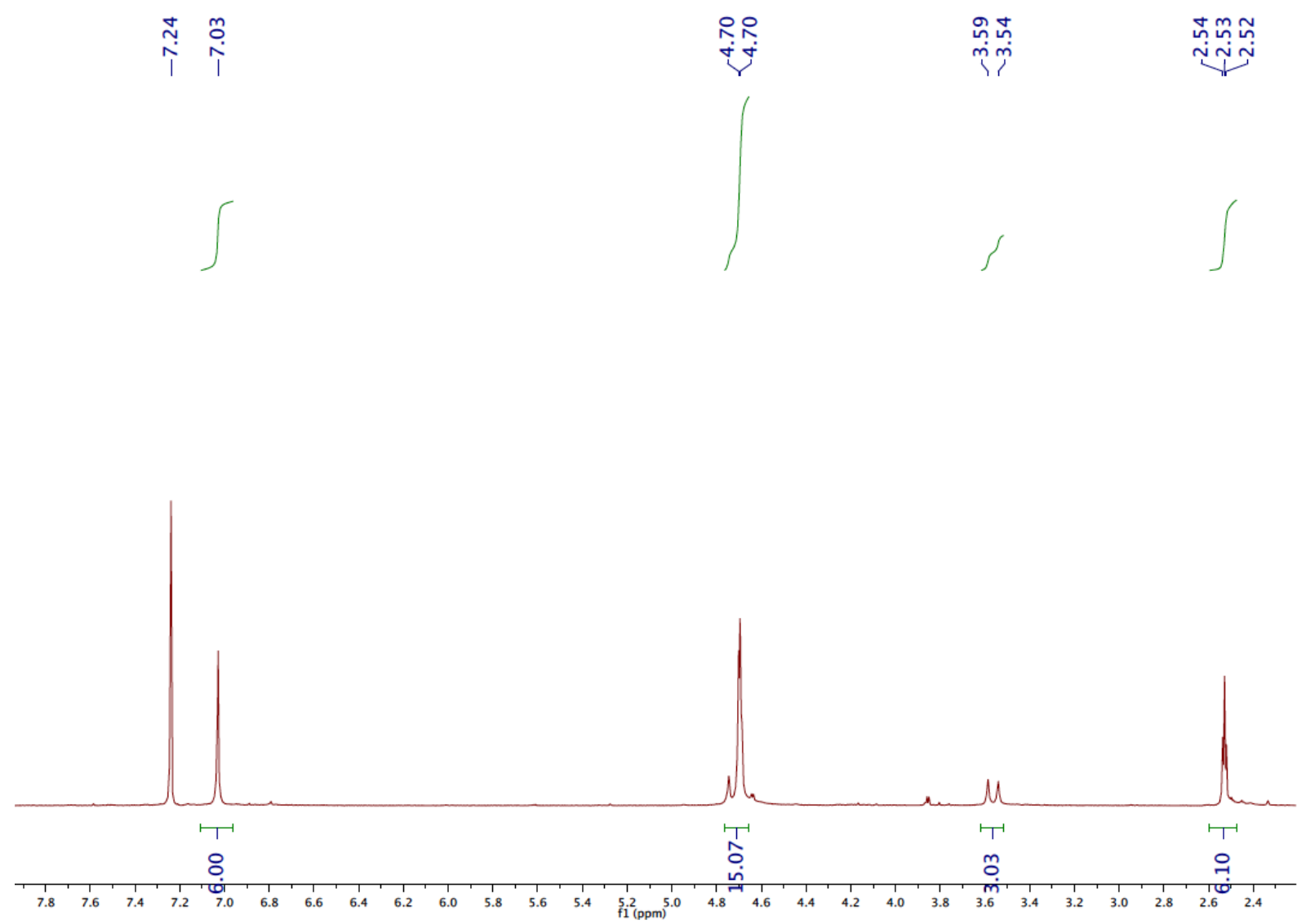

Figure S8: ${ }^{1} \mathrm{H}$ NMR spectrum of $5\left(300 \mathrm{MHz}, \mathrm{CDCl}_{3}\right)$ 


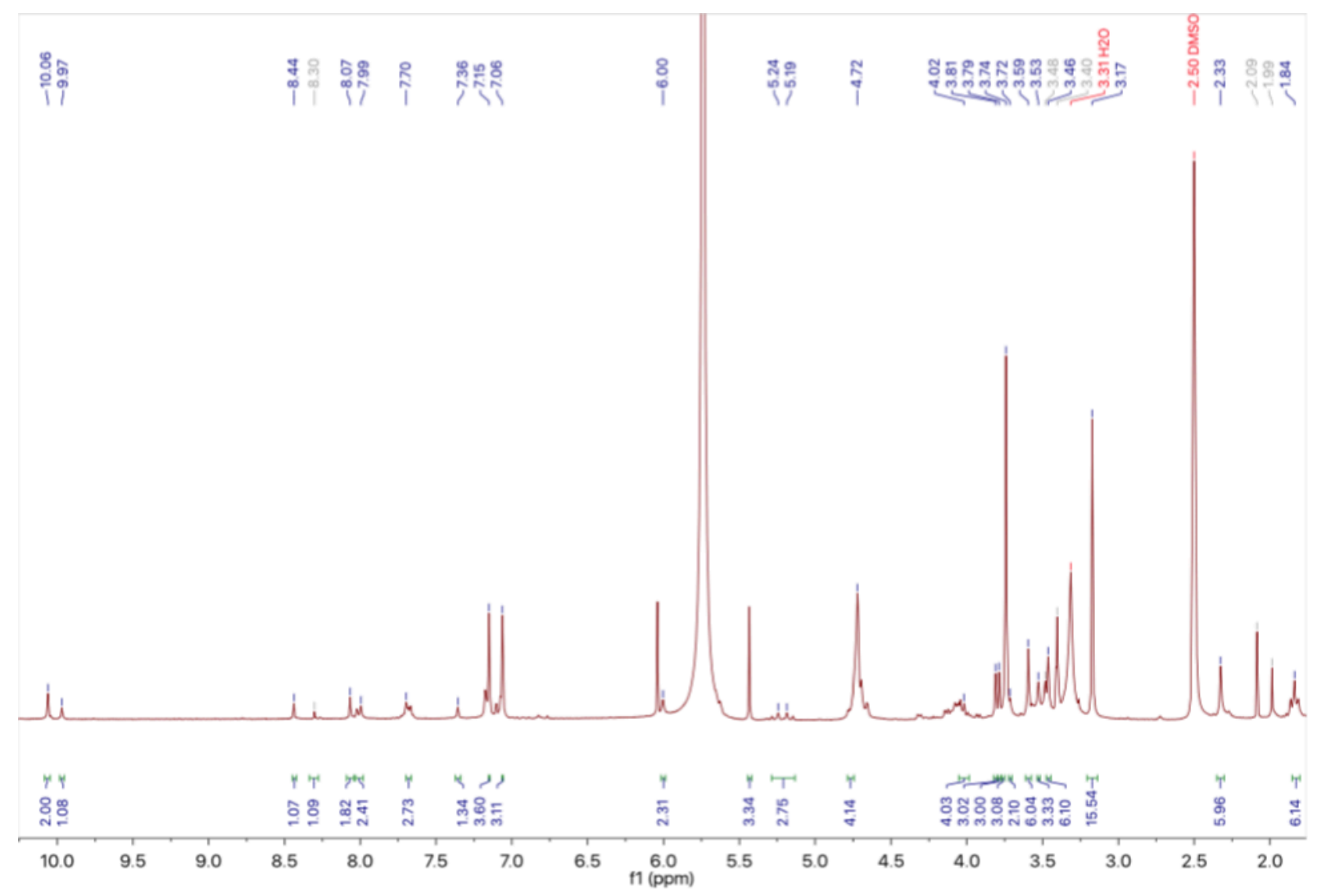

Figure S9: ${ }^{1} \mathrm{H}$ NMR spectrum of 1 PZn $\left(300 \mathrm{MHz}, \mathrm{DMSO}^{-\mathrm{d}_{6}}\right)$ 


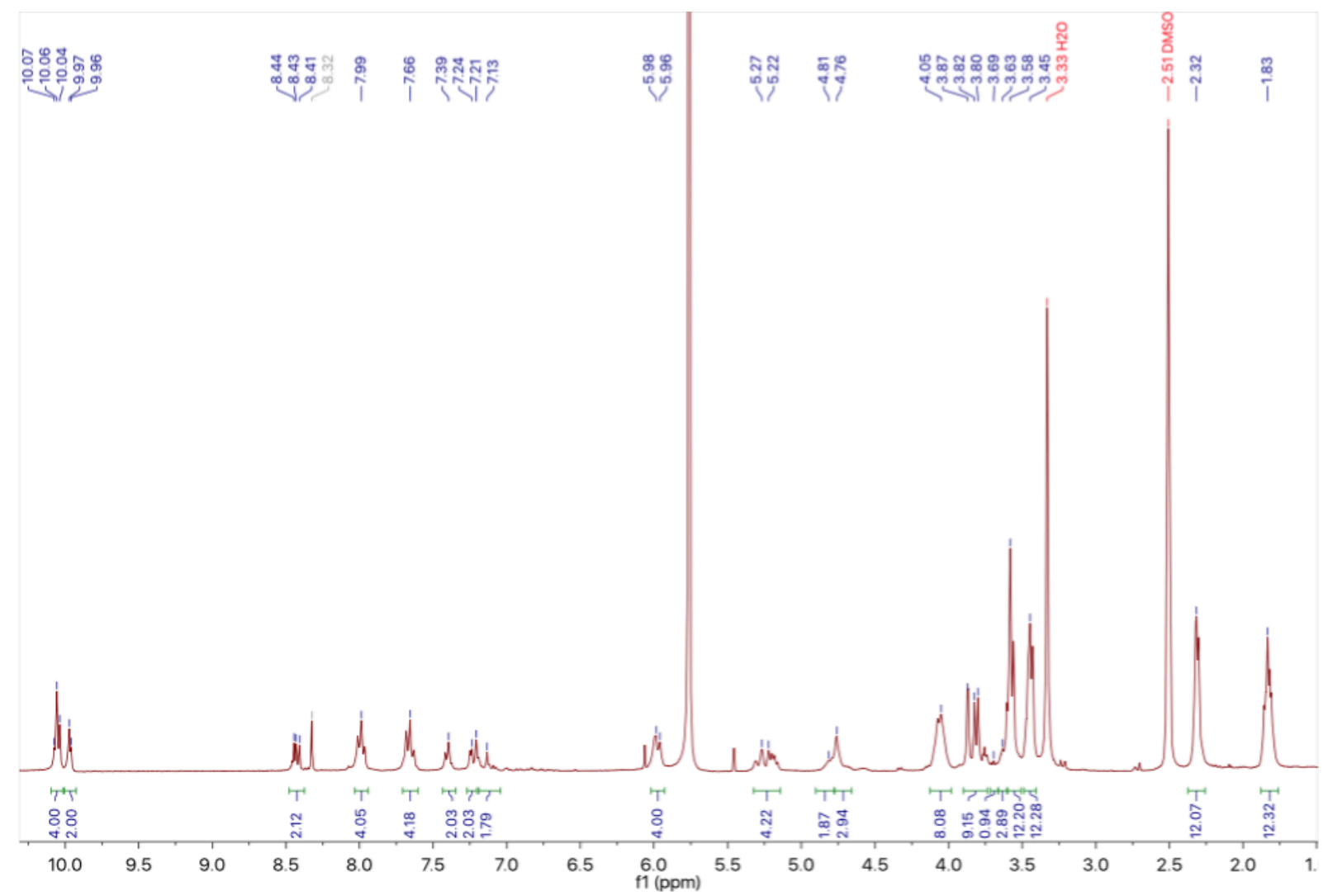

Figure S10: ${ }^{1} \mathrm{H}$ NMR spectrum of 2 PZn $\left(300 \mathrm{MHz}, \mathrm{DMSO}^{-} \mathrm{d}_{6}\right)$ 


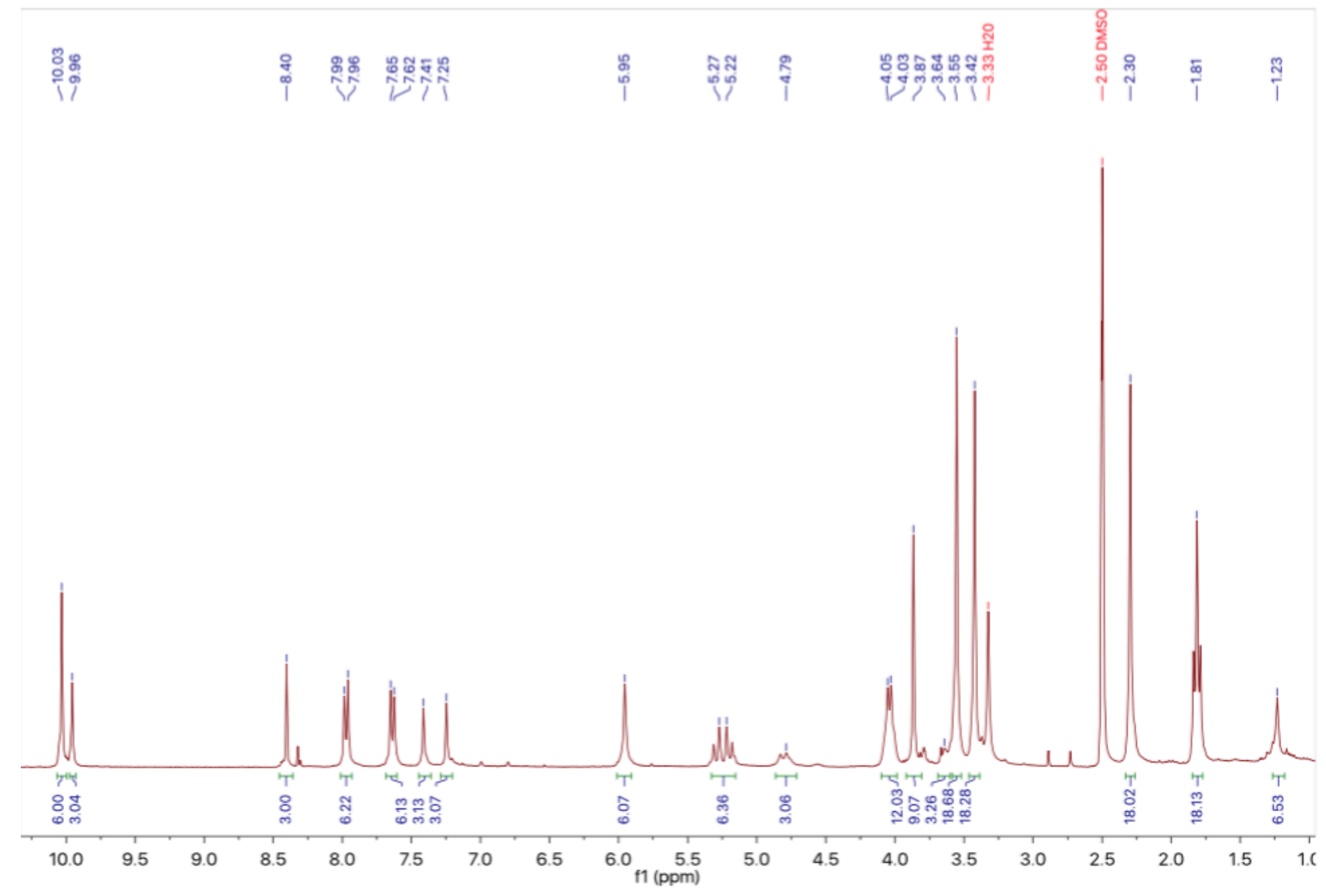

Figure S11 : ${ }^{1} \mathrm{H}$ NMR spectrum of 3PZn (300 MHz, DMSO-d $\left.\mathrm{d}_{6}\right)$ 


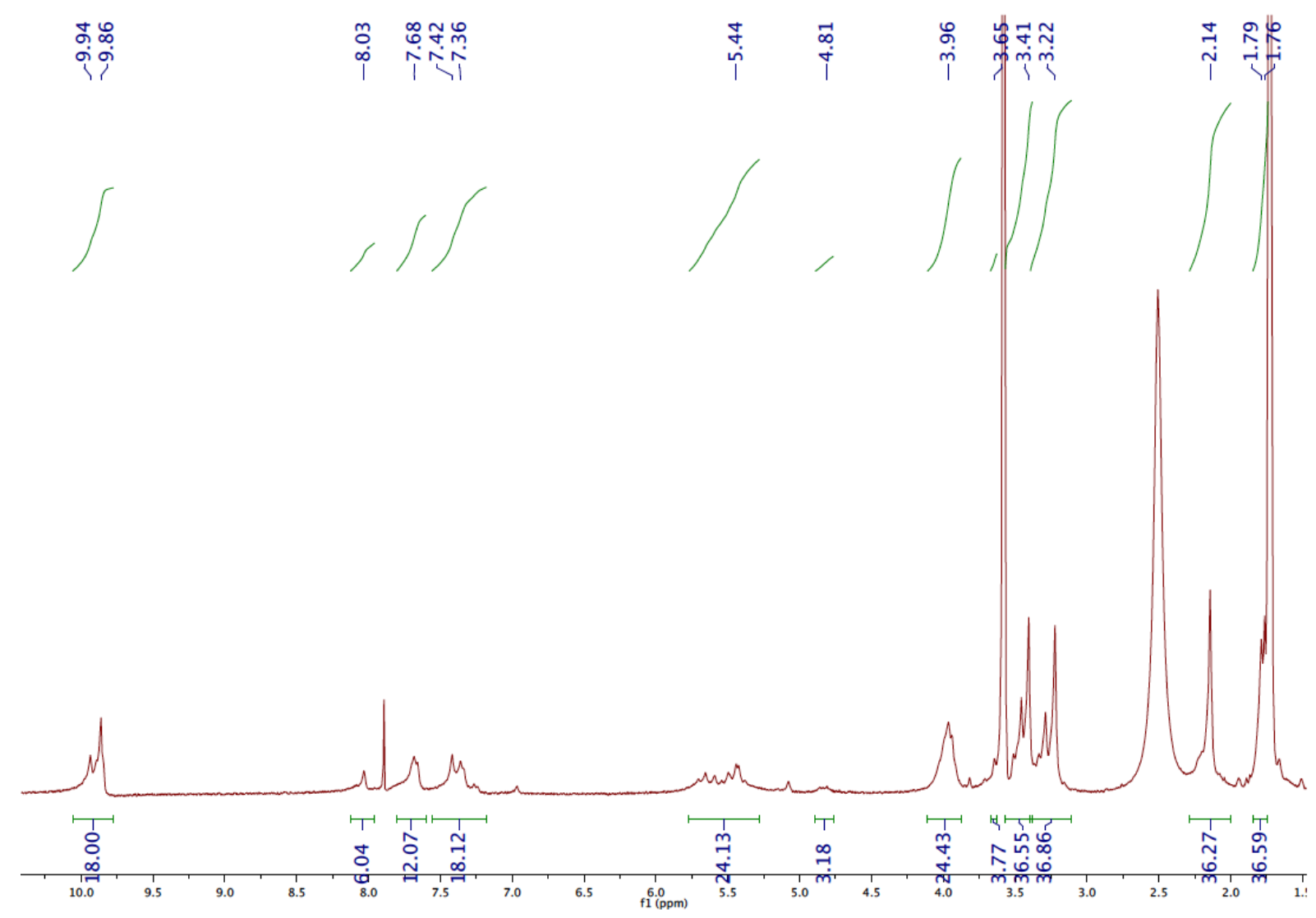

Figure S12 : ${ }^{1} \mathrm{H}$ NMR spectrum of 6PZn $\left(300 \mathrm{MHz}, \mathrm{THF}-\mathrm{d}_{8}\right)$ 


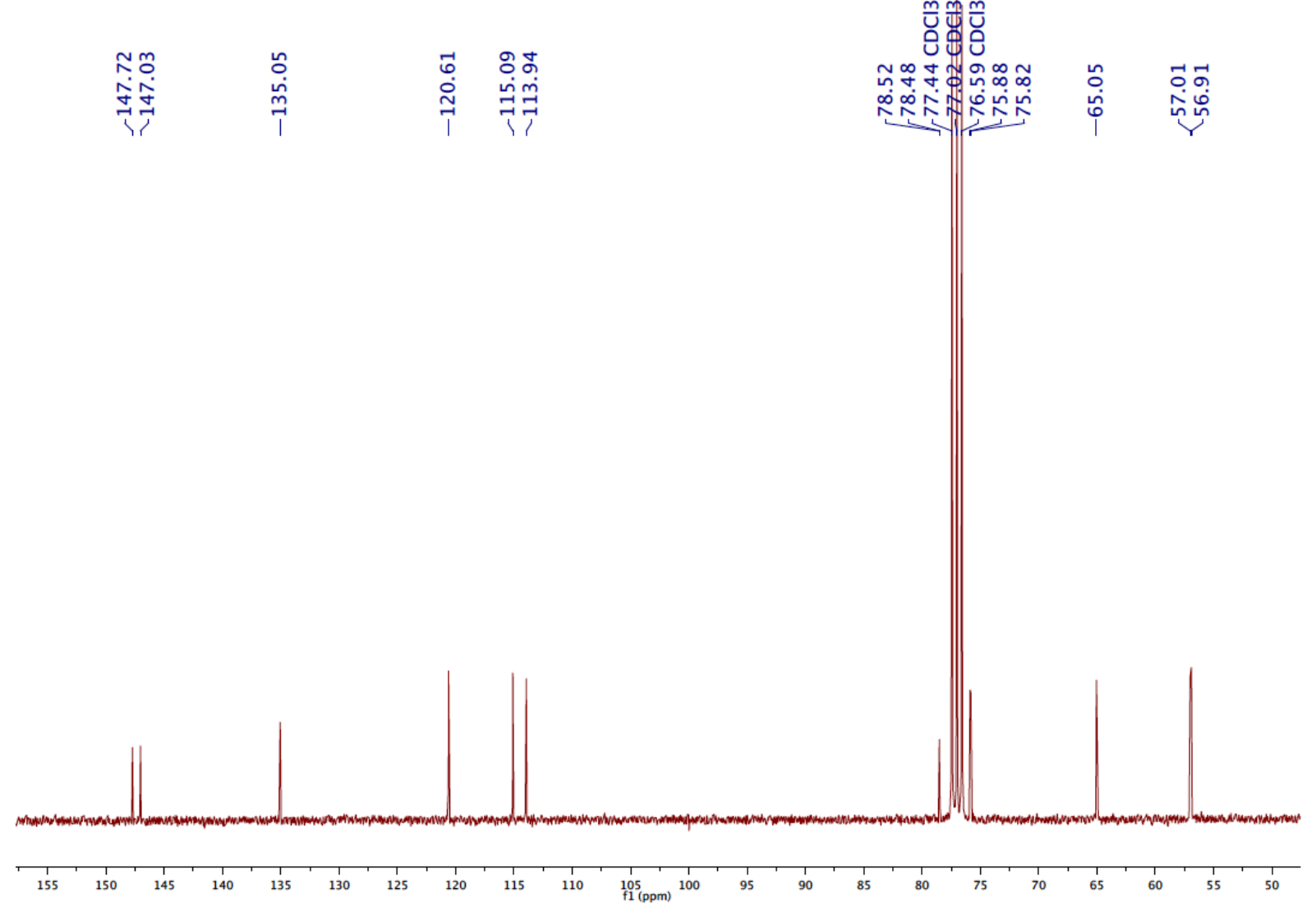

Figure S13 : ${ }^{13} \mathrm{C}$ NMR spectrum of $4\left(75 \mathrm{MHz}, \mathrm{CDCl}_{3}\right)$ 


\begin{tabular}{|c|c|c|c|}
\hline 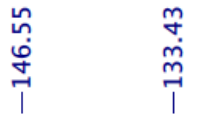 & $\begin{array}{l}\stackrel{0}{h} \\
\stackrel{1}{7} \\
1\end{array}$ & 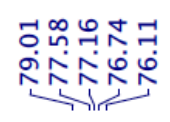 & 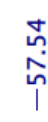 \\
\hline
\end{tabular}

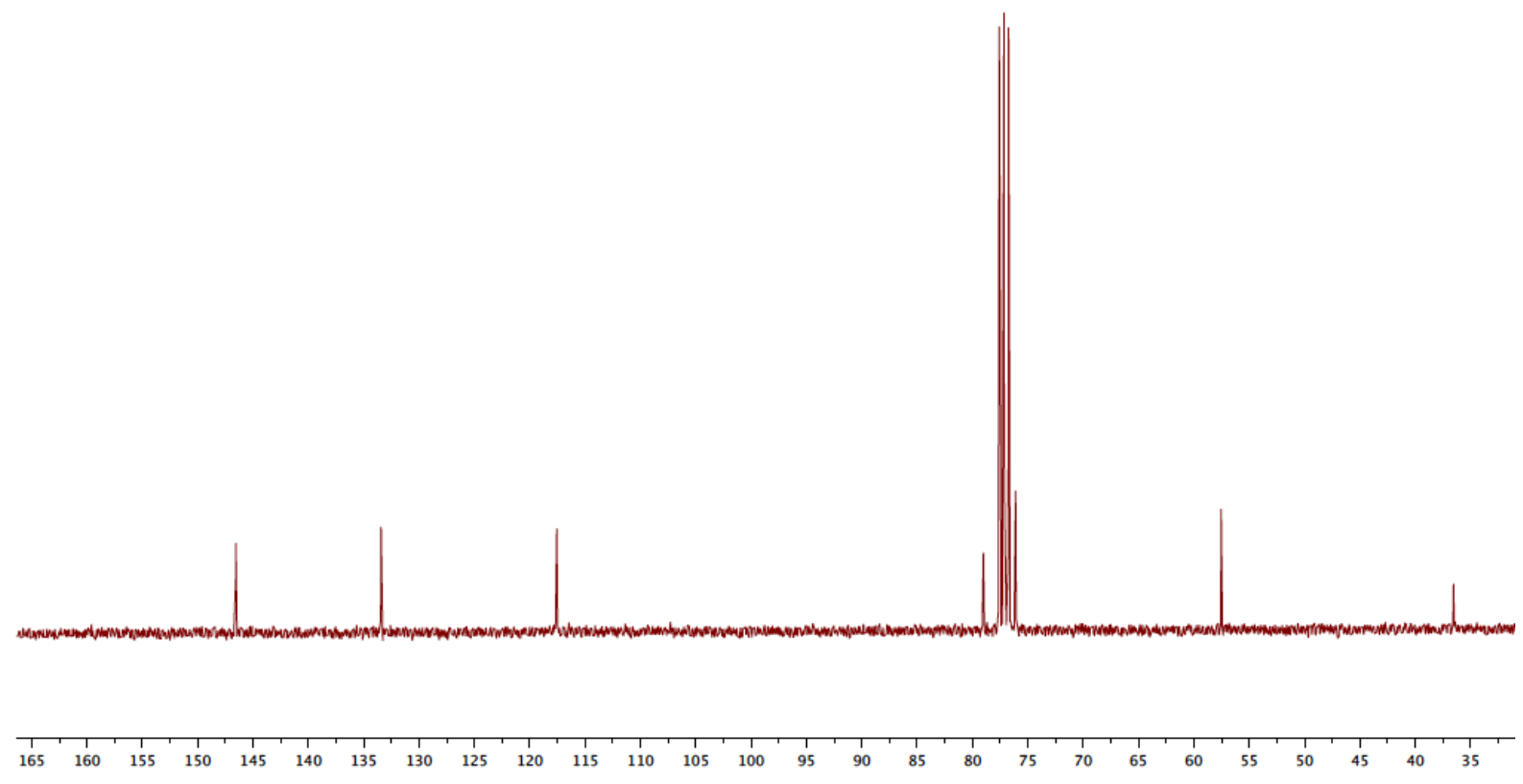

Figure S14 : ${ }^{13} \mathrm{C}$ NMR spectrum of $5\left(75 \mathrm{MHz}, \mathrm{CDCl}_{3}\right)$ 


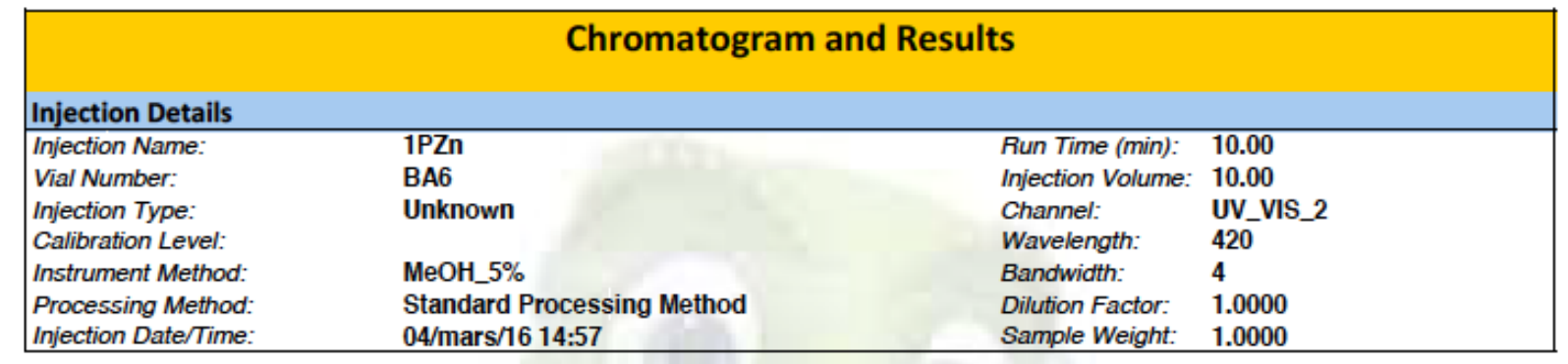

\section{Chromatogram}

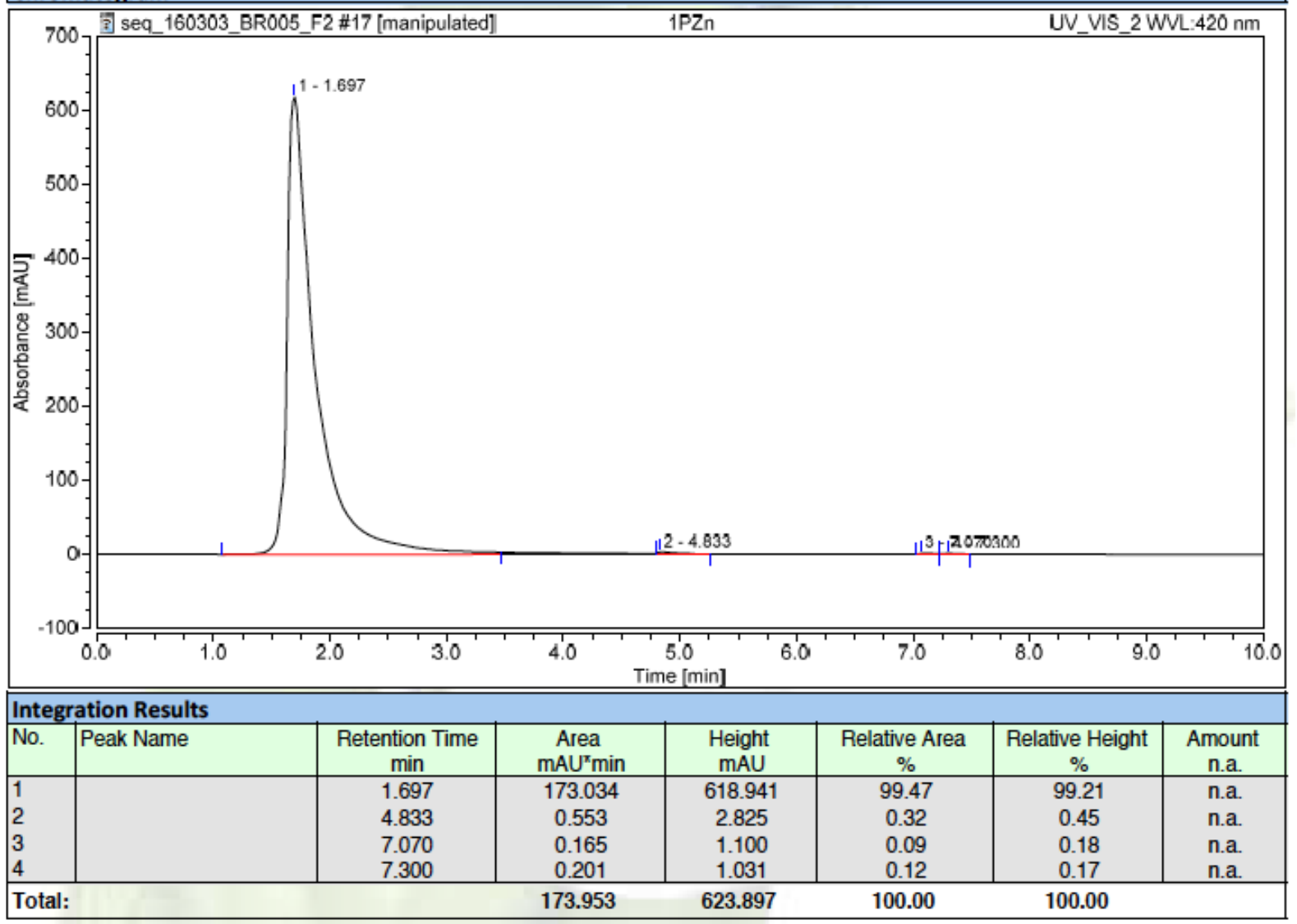

Figure S15: Analytical HPLC of 1PZn 


\begin{tabular}{|c|c|c|c|}
\hline \multicolumn{4}{|c|}{ Chromatogram and Results } \\
\hline $\begin{array}{l}\text { Injection Name: } \\
\text { Vial Number: } \\
\text { Injection Type: } \\
\text { Calibration Level: } \\
\text { Instrument Method: } \\
\text { Processing Method: } \\
\text { Injection Date/Time: }\end{array}$ & $\begin{array}{l}2 \text { PZn } \\
\text { BA7 } \\
\text { Unknown } \\
\text { MeOH_5\% } \\
\text { Standard Processing Method } \\
04 / \text { mars/16 15:08 }\end{array}$ & $\begin{array}{l}\text { Run Time (min): } \\
\text { Injection Volume: } \\
\text { Channel: } \\
\text { Wavelength: } \\
\text { Bandwidth: } \\
\text { Dilution Factor: } \\
\text { Sample Weight: }\end{array}$ & $\begin{array}{l}10.00 \\
10.00 \\
\text { UV_VIS_2 } \\
420 \\
4 \\
1.0000 \\
1.0000\end{array}$ \\
\hline
\end{tabular}

Chromatogram

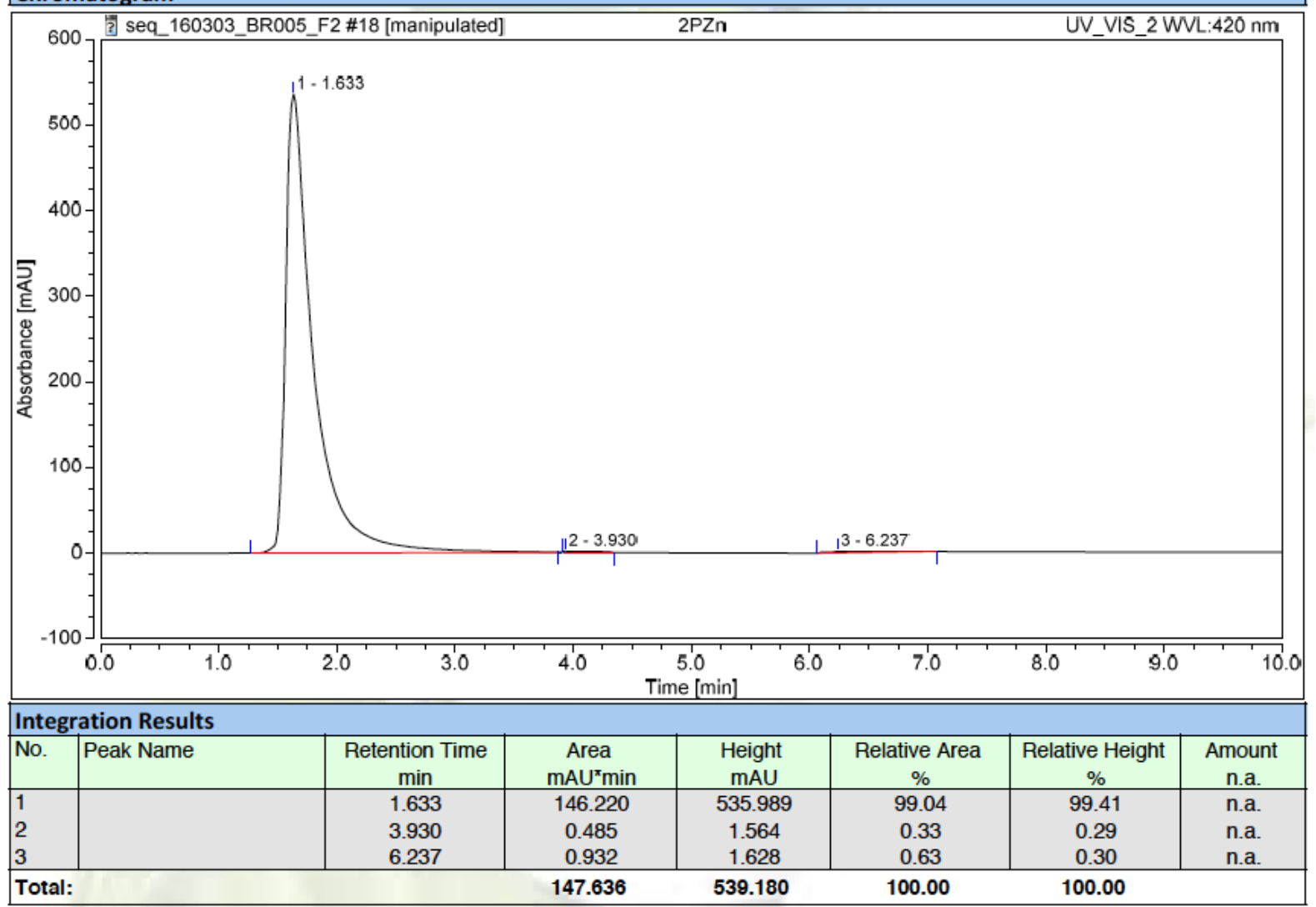

Figure S16: Analytical HPLC of 2PZn 


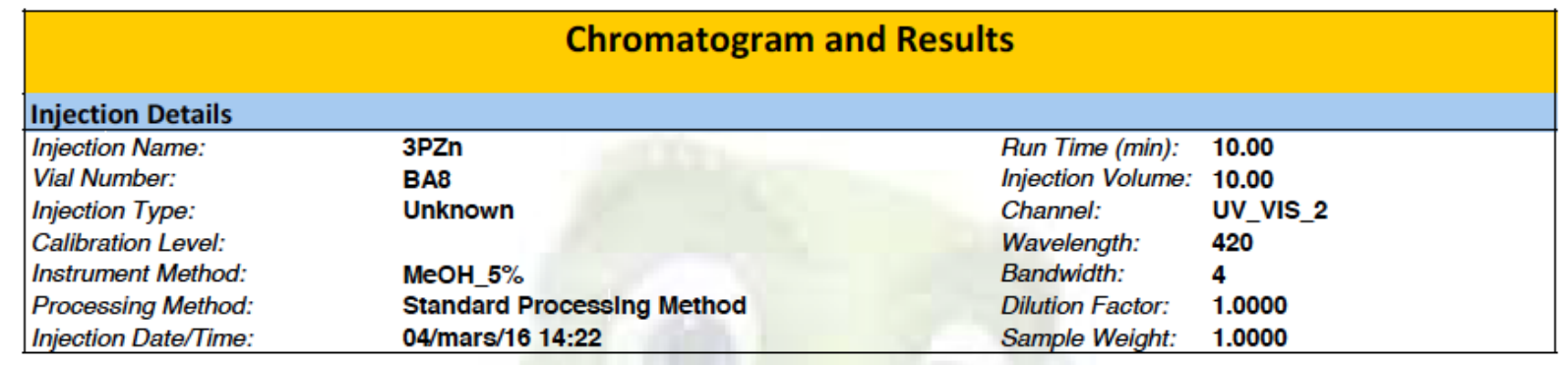

Chromatogram

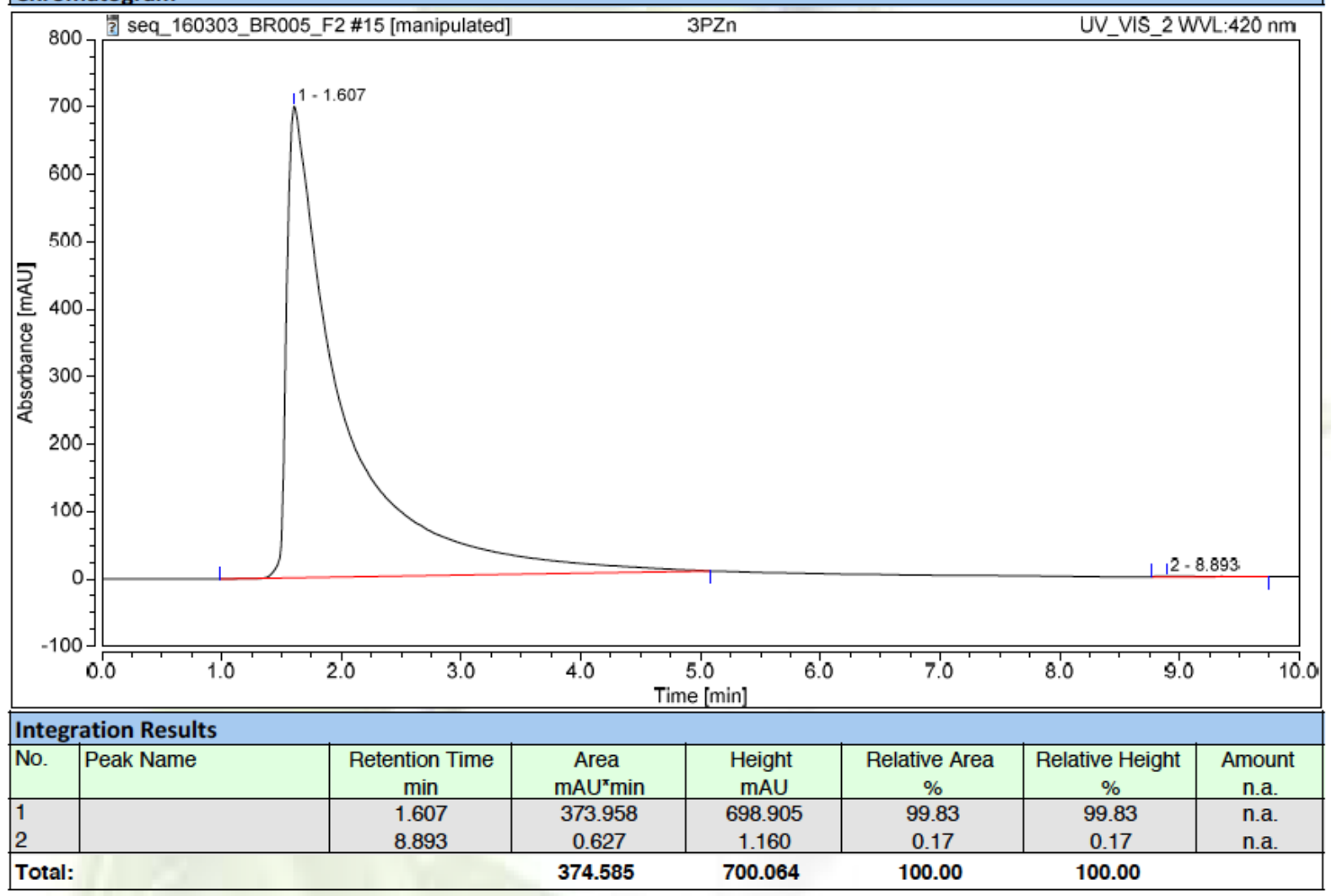

Figure S17: Analytical HPLC of 3PZn 


\section{Chromatogram and Results}

Injection Details

Injection Name:

Vial Number:

BR005_F2

BA6

Injection Type:

Unknown

Run Time (min):

10.00

Calibration Level:

MeOH_5\%

Injection Volume: 10.00

Instrument Method:

Processing Method:

Standard Processing Method

Channel:

UV_VIS

Wavelength:

420

Injection Date/Time. 03/mars/16 10:57

Bandwidth: 4

Dilution Factor: $\quad \mathbf{1 . 0 0 0 0}$

Sample Weight: 1.0000

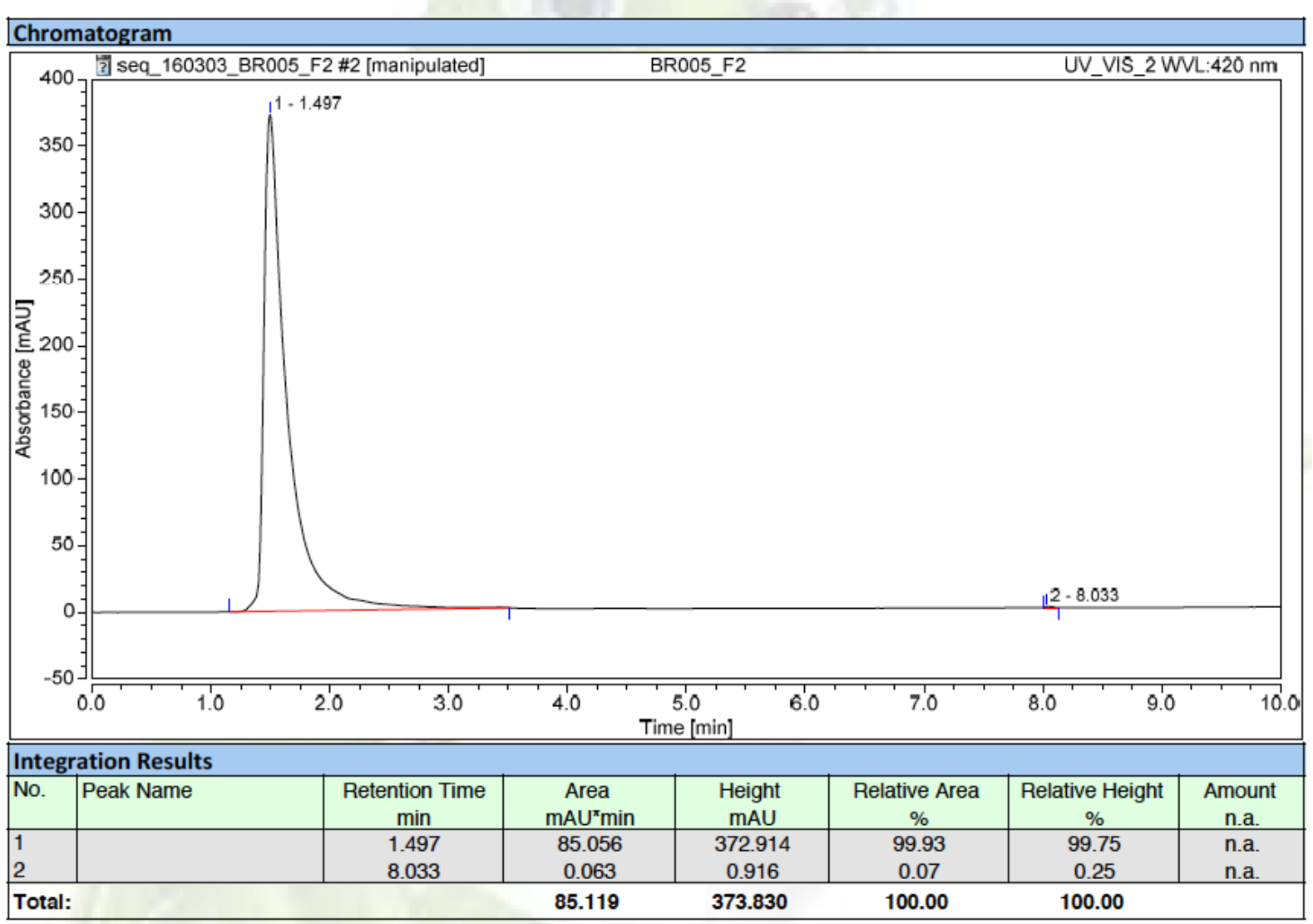

Figure S18: Analytical HPLC of 6PZn 

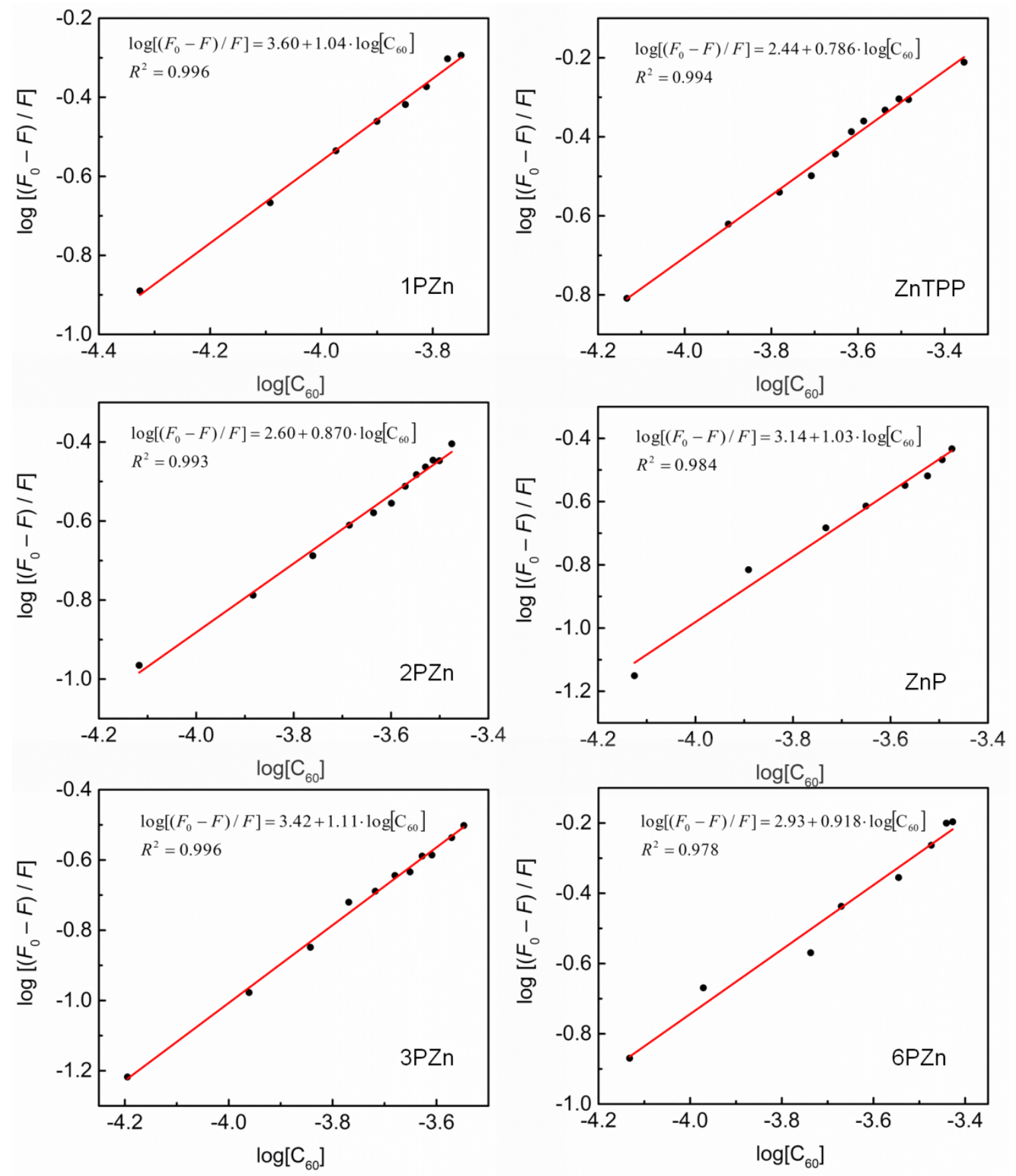

Figure S19. Graphs reporting $\log \left[\left(\mathrm{F}_{0}-\mathrm{F}\right) / \mathrm{F}\right]$ vs $\log \left[\mathrm{C}_{60}\right]$ (note that $\mathbf{Z n P}=\mathbf{3}$ ). 


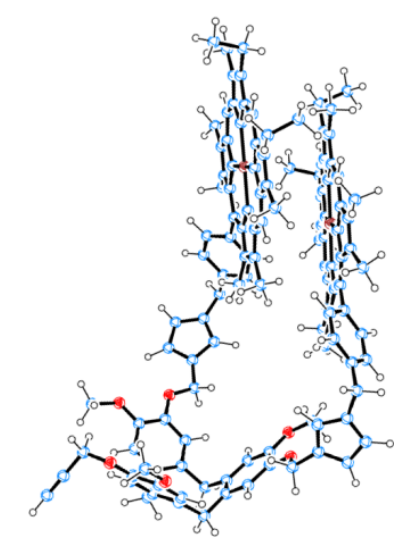

$331.25 \mathrm{kcal} / \mathrm{mol}$

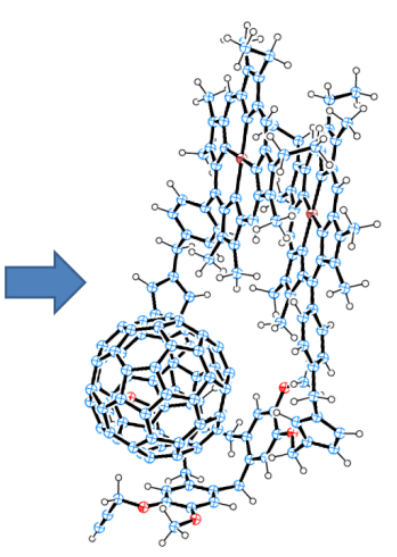

$2111.31 \mathrm{kcal} / \mathrm{mol}$

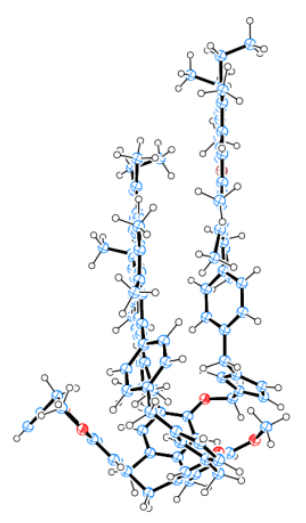

$325.29 \mathrm{kcal} / \mathrm{mol}$

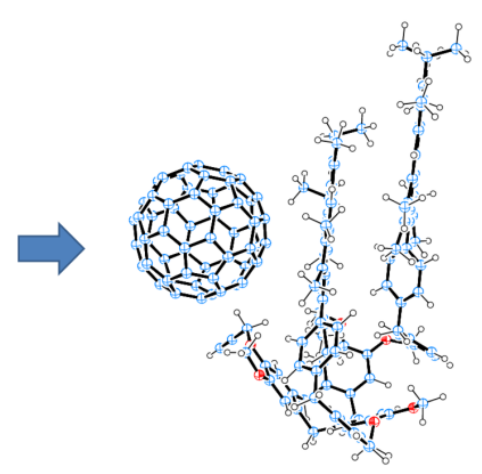

$2110.93 \mathrm{kcal} / \mathrm{mol}$

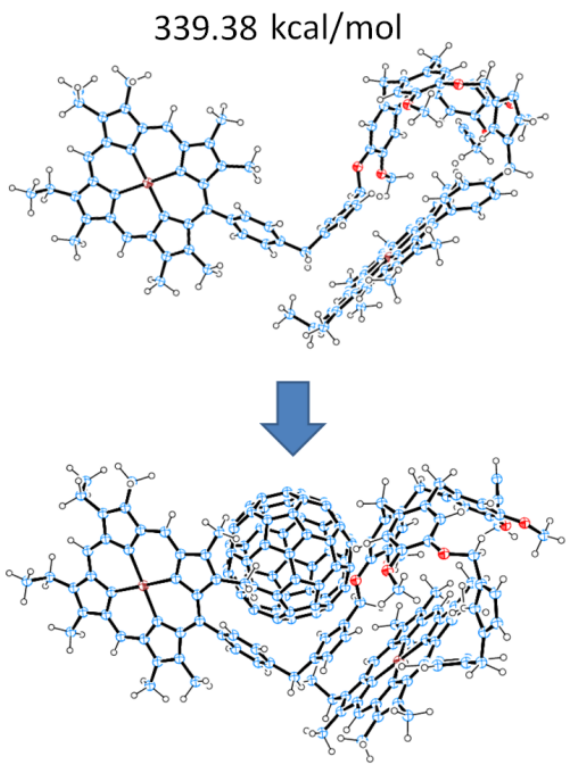

$2122.42 \mathrm{kcal} / \mathrm{mol}$

$349.73 \mathrm{kcal} / \mathrm{mol}$
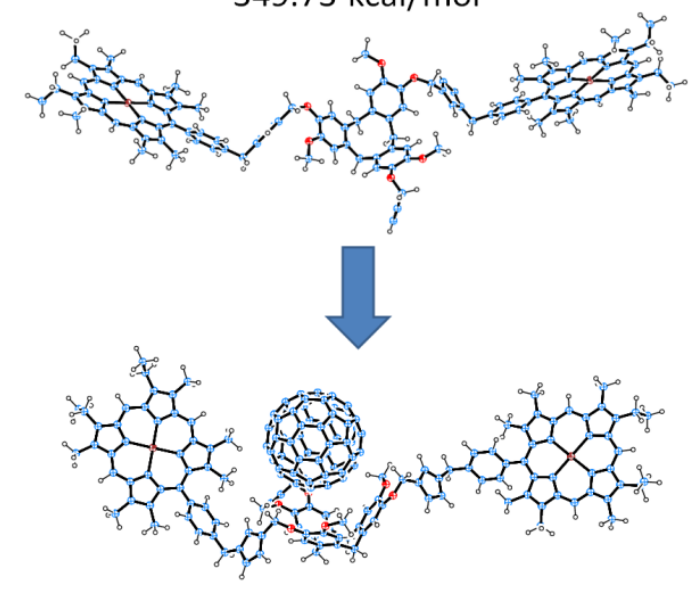

$2130.87 \mathrm{kcal} / \mathrm{mol}$

This image above is from Figure 8.

Figure S20. Computer modeling of $\mathbf{2} \mathbf{P Z n}$ and corresponding $\mathbf{2} \mathbf{P Z n} \cdots \mathrm{C}_{60}$ in some upper energy conformations. 

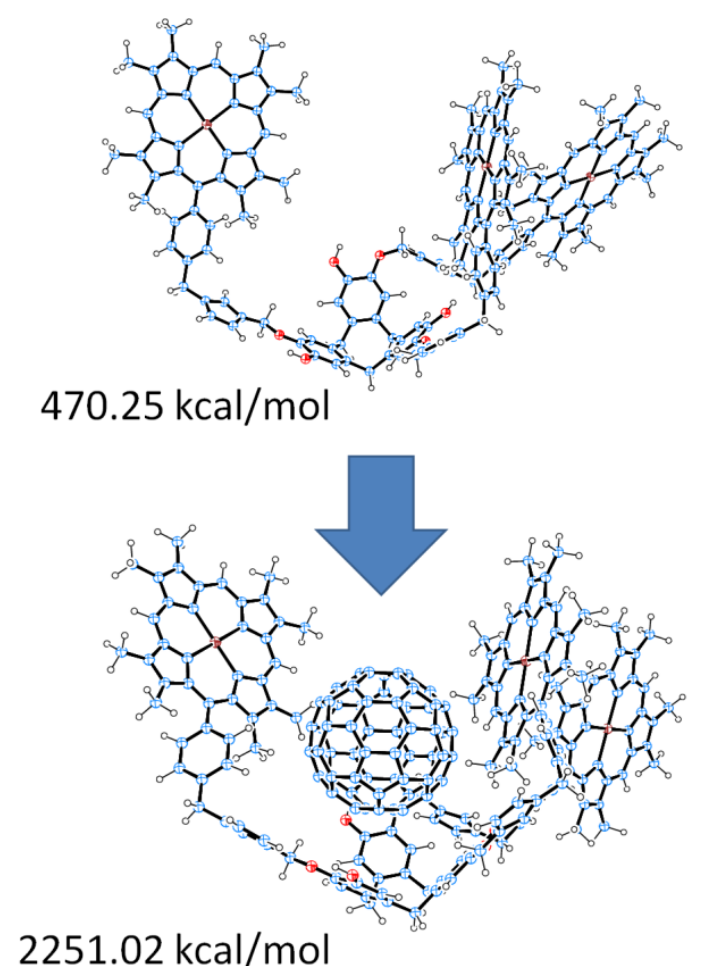

Figure S21. Computer modeling of $\mathbf{3 P Z n}$ and corresponding $\mathbf{3 P Z n} \cdots \mathrm{C}_{60}$ in one upper energy conformation. 